\title{
Characterization of Anthraquinone-2-Carbonyl Chloride as an Alcohol Derivatization Reagent for Negative Ion Chemical Ionization Mass Spectrometry
}

\author{
J. T. Simpson, D. S. Torok, R. Worman, B.J. Quearry, and S. P. Markey \\ Section on Analytical Biochemistry, National Institute of Mental Health, National Institutes of Health, \\ Bethesda, Maryland, USA
}

\section{H. Ziffer}

Laboratory of Chemical Physics, National Institute of Diabetes and Digestive and Kidney Diseases, National Institutes of Health, Bethesda, Maryland, USA

\begin{abstract}
Anthraquinone-2-carbonyl chloride has been utilized as a derivatization reagent for alcohols to impart electron affinity and aid in transport via a particle beam liquid chromatography-mass spectrometry (LC/MS) interface. In addition, the gas chromatographic-mass spectrometry, UV, fluorescence, and electrochemical characteristics of the derivatives were determined. A series of model compounds, 2-phenylethanol (phenethyl alcohol), 1-phenyl-2-propanol, 2methyl-1-phenyl-2-propanol, hexanol, and methyl 2-methylglycerate, were used as analytes.

The particle beam LC/MS properties of the resultant anthraquinone carboxylate esters were determined in electron impact (EI) and negative ion chemical ionization (NCI) modes. The NCI responses of these anthraquinone carboxylate esters were compared with the corresponding 3,5-dinitrobenzoate esters. The anthraquinone carboxylate esters exhibited an $\mathrm{NCI}$ to EI sensitivity enhancement of 113 and were detected in $\mathrm{NCI}$ at a tenfold lower concentration than the corresponding 3,5-dinitrobenzoate esters. A detection limit of $26 \mathrm{pg}$ injected on column was achieved for phenethyl anthraquinone carboxylate in NCI by using selected ion monitoring. (J Am Soc Mass Spectrom 1995, 6, 148-151)
\end{abstract}

$\mathrm{T}$ The sensitivity enhancement that can be achieved by the use of negative ion chemical ionization (NCI) in mass spectrometry is well documented [1-4]. Although some halogenated or highly conjugated molecules have inherent electron affinity, most compounds of biological interest first must be derivatized to impart the ability to capture electrons. An extensive literature on NCI derivatization techniques suitable for amines or carboxylic acids has appeared and is summarized in several texts $[5,6]$. Alcohols remain one compound class for which there are a limited number of electron capturing derivatives that produce stable molecular anions. To this end, we have investigated the use of anthraquinone-2-carbonyl chloride $(\mathrm{ACCl})$ as a reagent for the derivatization of alcohols with subsequent analysis by particle beam (PB) liquid chromatography-negative chemical ionization mass spectrometry (LC-NCI/MS).

$\mathrm{ACCl}$ has been used for the labeling of polymers [7] to impart a UV chromophore and to label steroids and

Address reprint requests to S. P. Markey, National Institutes of Health/National Institute of Mental Health, Building 10, Room 3D40, 10 Center Drive, MSC 1262, Bethesda, MD 20892-1262. aliphatic alcohols to produce dyes [8]. Our interest in its use as a reagent for negative ion chemical ionization mass spectrometry ( $\mathrm{NCI} / \mathrm{MS}$ ) stems from the observation that 1-methylaminoanthraquinone (MAAQ) is an excellent sensitivity standard for particle beam LC-NCI/MS [9]. MAAQ has good transport properties and is detectable at $1 \mathrm{pg}$ in NCI single ion monitoring experiments that use direct flow injection. Given the potential utility of the anthraquinone electrophore, it was thought that an anthraquinone acid chloride would be sufficiently reactive to derivatize alcohols and yield structurally significant ions in the NCI mass spectrum of the derivatives. ACCl was compared with 3,5-dinitrobenzoyl chloride in terms of reactivity and $\mathrm{NCI}$ response of the derivatives formed. 3,5-Dinitrobenzoyl chloride was chosen for comparison because of its high electron capture cross section [6] and its low volatility, which should enhance transport of the derivatives through the PB interface.

Several model compounds were used as analytes to evaluate ACCl: these include 2-phenylethanol (phenethyl alcohol), 1-phenyl-2-propanol, 2-methyl-1phenyl-2-propanol, hexanol, and methyl 2-methylglycerate. The substituted phenyl alcohols were chosen 
to allow monitoring of reaction progress by highperformance liquid chromatography (HPLC) with UV detection and to probe the reactivity of $\mathrm{ACCl}$ with $1^{\circ}$ (phenethyl alcohol), $2^{\circ}$ (1-phenyl-2-propanol), and $3^{\circ}$ (2-methyl-1-phenyl-2-propanol) alcohols. Phenethyl alcohol is a component of several essential oils, including rose, carnation, hyacinth, and orange blossom, and is a prominent component of rose perfumes [10]. Methyl 2-methylglycerate is produced from thymine glycol by sodium borohydride reduction and is being studied as a potential marker for DNA damage [11]. This ester is a cis-glycol that contains a primary and sterically hindered tertiary alcohol.

We report here the synthesis, reactivity, and sensitivity of $\mathrm{ACCl}$ as a reagent for derivatization of alcohols. Furthermore, the UV, fluorescence, electrochemical, and gas chromatographic properties of the derivatives are reported.

\section{Experimental Section}

\section{Synthesis of Anthraquinone-2-carbonyl Chloride}

Anthraquinone-2-carboxylic acid (Aldrich Chemical, Milwaukee, WI; $2.52 \mathrm{~g}, 10 \mathrm{mmol}$ ) in thionyl chloride (Aldrich; $125 \mathrm{~mL}$ ) was refluxed for $18 \mathrm{~h}$ under nitrogen. The thionyl chloride was removed from the clear solution by atmospheric distillation to give a yellow solid, which was recrystallized from hot benzene (50 $\mathrm{mL}$ ) by the addition of hexane $(75 \mathrm{~mL})$ followed by cooling to afford the carbonyl chloride $(2.0 \mathrm{~g}, 74 \%): \mathrm{mp}$ 145-146 ${ }^{\circ} \mathrm{C} ;{ }^{1} \mathrm{H}$ NMR ( $d_{6}$-acetone) $\delta 8.00(\mathrm{~m}, 2 \mathrm{H}), 8.32$ $(\mathrm{m}, 2 \mathrm{H}), 8.48(\mathrm{~d}, J=8.2 \mathrm{~Hz}, 1 \mathrm{H}), 8.59(\mathrm{~d}, J=9.1 \mathrm{~Hz}$, 1H), $8.92(\mathrm{~s}, 1 \mathrm{H}) ;{ }^{13} \mathrm{C} \mathrm{NMR}\left(\mathrm{CDCl}_{3}\right) \delta 127.4(\mathrm{CH}), 127.5$ $(\mathrm{CH}), 128.0(\mathrm{CH}), 130.3(\mathrm{CH}), 133.0(\mathrm{C}), 133.7(\mathrm{C}), 134.6$ $(\mathrm{CH}), 134.7(\mathrm{CH}), 135.1(\mathrm{CH}), 137.1(\mathrm{C}), 137.6(\mathrm{C}), 167.3$ (C(O)Cl), $181.4(\mathrm{CO}), 181.8(\mathrm{CO})$.

2-Phenylethanol, 1-phenyl-2-propanol, 2-methyl-1phenyl-2-propanol, 4-dimethylaminopyridine and hexanol were obtained from Aldrich Chemical Co. and used as supplied. 3,5-Dinitrobenzoyl chloride was obtained from Eastman Chemicals (Rochester, New York). The synthesis of the methyl 2-methylglycerate has been described previously [11]. $\left({ }^{2} \mathrm{H}_{3}\right)$ Methyl 2-methylglycerate was produced from 2-methylglyceric acid [12] by esterification with deuterated methanol (Aldrich) in the presence of $\mathrm{HCl}$. All solvents were of HPLC grade.

\section{Derivatization}

An aliquot of an acetonitrile solution of alcohol (100 $\mu \mathrm{L}, \sim 200 \mathrm{ng} / \mu \mathrm{L})$ was added to $50 \mu \mathrm{L}$ of a $1 \%$ solution of acid chloride in acetonitrile and $50 \mu \mathrm{L}$ of a $0.5 \%$ solution of dimethylaminopyridine (DMAP) in acetonitrile. The molar ratio of alcohol:ACCl:DMAP was approximately 1:10:10. The mixture was then heated at $70{ }^{\circ} \mathrm{C}$ for $20 \mathrm{~min}$ and analyzed.

\section{Standard Curve}

A standard curve was constructed for methyl 2-methylglycerate by using $\left({ }^{2} \mathrm{H}_{3}\right)$ methyl 2-methylglycerate as an internal standard. Standards were derivatized and 2 $\mu \mathrm{L}$ (of $50 \mu \mathrm{L}$ total) were injected with selected ion monitoring (SIM) of the molecular anions $(m / z 368$ and 371) of analyte and internal standard, respectively. The chromatographic peaks were then integrated and area ratios were calculated.

\section{Mass Spectrometry and Chromatography}

Particle beam liquid chromatography-mass spectrometry (LC/MS) analyses were performed on a HewlettPackard (Palo Alto, CA) 5989 mass spectrometer interfaced with a HP-1050 HPLC via a HP-59980B particle beam interface. The ion source temperature was 300 ${ }^{\circ} \mathrm{C}$. The desolvation chamber was held at $55^{\circ} \mathrm{C}$ and the nebulization pressure was $60 \mathrm{lb} / \mathrm{in}^{2}$. Full scan data were recorded from $m / z 60$ to 650 . Gas chromatography-mass spectrometry (GC/MS) analyses were performed on a Finnigan-Mat (San Jose, CA) TSQ-70 (scanning Q1) interfaced with a Varian 3400 gas chromatograph (Varian Associates, Walnut Creek, CA). Ion source temperature was $200{ }^{\circ} \mathrm{C}$. Methane was used as reagent gas for all chemical ionization experiments.

HPLC separations were performed on a $100 \times 2.1$ mm Hewlett Packard ODS Hypersil C18 column, 5- $\mu \mathrm{m}$ particle size, with a linear gradient from $50: 5050-\mathrm{mM}$ ammonium acetate $\left(\mathrm{NH}_{4} \mathrm{OAc}\right) / \mathrm{CH}_{3} \mathrm{CN}$ to $100 \%$ $\mathrm{CH}_{3} \mathrm{CN}$ over $15 \mathrm{~min}$, holding at $100 \%$ for $5 \mathrm{~min}$. The flow rate was $0.3 \mathrm{~mL} / \mathrm{min}$. UV data were obtained with a HP-1050 diode array detector scanning from 200 to $600 \mathrm{~nm}$. Fluorescence data were obtained with a HP-1046 fluorescence detector. Electrochemical detection was performed with a Waters (Milford, MA) 460 electrochemical detector. To minimize peak broadening in LC/MS data acquisition, other in-line detectors were not used.

Gas chromatography (GC) separations were performed on a HP-1, $12-\mathrm{m} \times 0.2-\mathrm{mm}$ capillary column, $0.33-\mu \mathrm{m}$ film thickness. The oven was ramped from 70 to $300{ }^{\circ} \mathrm{C}$ at $20{ }^{\circ} \mathrm{C} / \mathrm{min}$, with the injection port at 300 ${ }^{\circ} \mathrm{C}$.

\section{Results and Discussion}

$\mathrm{ACCl}$ is a stable crystalline compound that can be stored conveniently at room temperature. It has limited solubility in $\mathrm{CH}_{3} \mathrm{CN}$ and was used as a saturated solution in these experiments. It was found to have good solubility in methyl isobutyl ketone (MIBK), and its use with toluene also has been reported [8]. Both MIBK and toluene coeluted with the alcohols being monitored, whereas $\mathrm{CH}_{3} \mathrm{CN}$ was a component of the liquid chromatography (LC) solvent gradient and consequently was very convenient for reaction monitoring. 
The anthraquinone carboxylate derivatives formed under mild conditions in good yields $(92 \%)$ with the primary alcohols studied. A secondary alcohol, 1phenyl-2-propanol, also reacted with lower yields (30\%). Derivatives of tertiary alcohols did not form in detectable quantities. Yields were based on monitoring the response of the parent phenyl alcohol at $211 \mathrm{~nm}$. Several hindered bases, including diisopropylethyl amine, 1,8-diazobicyclo[5.4.0] undec-7-ene (DBU), pyridine, and triethyl amine were tested as catalysts. DMAP provided the greatest product yield. Further optimization of the reaction conditions may improve the yield for secondary alcohols.

The anthraquinone carboxylate derivatives of alcohols proved to be ideal for analysis by LC-NCI/MS via the particle beam interface. Low picogram amounts of these derivatives can be detected with on-line chromatography. In the $\mathrm{NCI}$ mass spectra of the $\mathrm{ACCl}$ derivatives all of the ion current is carried by the molecular anion and its isotope peak. This is advantageous for quantitation by SIM.

An example of the sensitivity of the derivatives is shown in Figure $1 \mathrm{a}$ and $\mathrm{b}$, where 256 and $26 \mathrm{pg}$, respectively, of phenethyl anthraquinone carboxylate were injected on-column, monitoring the molecular anion $m / z$ 356. The results of a similar SIM experiment with the anthraquinone carboxylate ester of
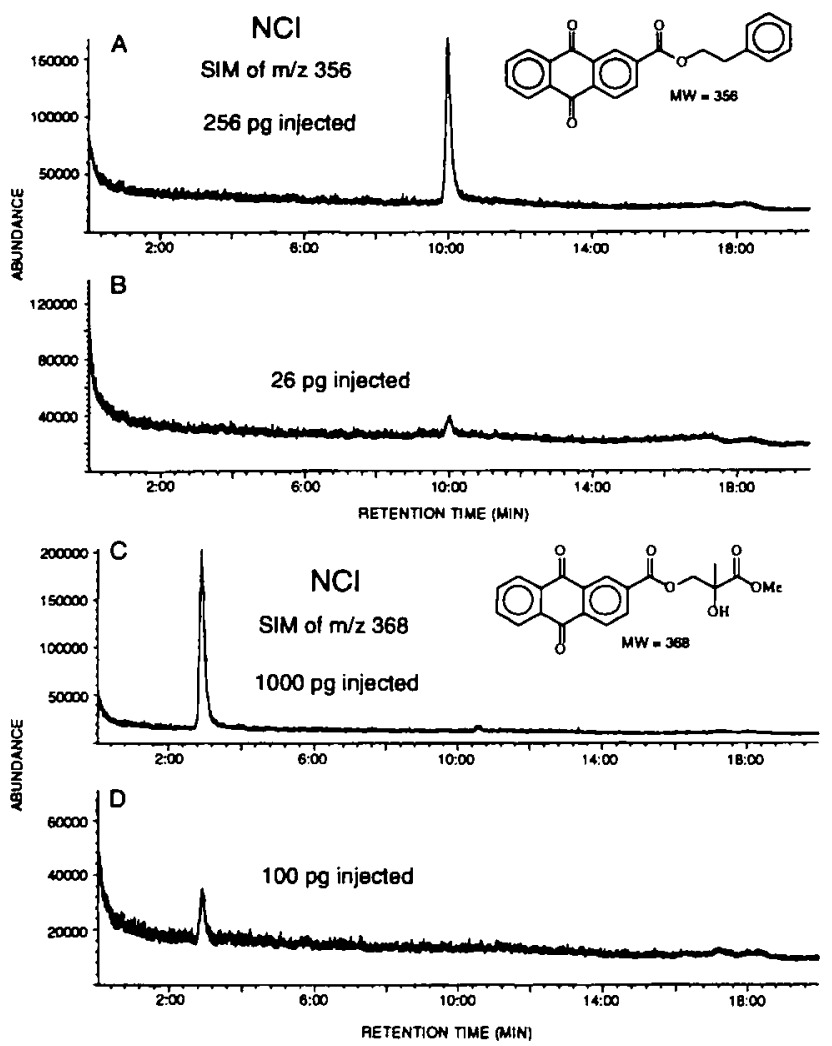

Figure 1. Particle beam-negative ion chemical ionization single ion chromatograms of phenethyl anthraquinone carboxylate (a) $256 \mathrm{pg}$ injected, (b) $26 \mathrm{pg}$ injected, and methyl 2-methylglycerate anthraquinone carboxylate (c) $1000 \mathrm{pg}$ injected, (d) $100 \mathrm{pg}$ injected. methyl 2-methylglycerate are shown in Figure 1c and $\mathrm{d}$. These limits of detection were achieved with diluted standards. The results of a standard curve showed a linear response for the anthraquinone carboxylate ester of methyl 2-methylglycerate over the range of 1-1000 $\mathrm{ng} /$ standard with a correlation coefficient of 0.99 .

The NCI mass spectra of the anthraquinone derivatives did not exhibit fragmentation, even at an ion source temperature of $300{ }^{\circ} \mathrm{C}$. In contrast, the NCI mass spectrum for phenethyl-3,5-dinitrobenzoate contained a prominent ion at $[\mathrm{M}-30]^{-}$that resulted from loss of NO.

The total ion signal in $\mathrm{NCI}$ for the anthraquinone esters was greater than 100 times that in electron ionization (EI). By comparison, the dinitrophenyl derivatives were a factor of 20 more sensitive in NCI versus EI. Although electron capture literature exists for both the anthraquinone [13,14] and dinitrophenyl electrophores [6], a direct comparison was not found. In this study the relative sensitivities of the anthraquinone and dinitrobenzoate esters were compared in $\mathrm{NCI}$ and the anthraquinone esters were found to be a factor of 10 more sensitive.

The total ion chromatograms of the anthraquinone reaction mixtures were relatively simple. In general, two components were observed: the expected ester and by-product anthraquinone carboxylic acid (AQ acid), which elutes early. This was typical of all the analytes derivatized with $\mathrm{ACCl}$. Depending on the retention time of the analyte derivative, the $A Q$ acid could easily be diverted from the mass spectrometer via a divert valve.

The use of $50-\mathrm{mM} \mathrm{NH}_{4} \mathrm{OAc}$ as the aqueous component of the gradient improved the signal of the anthraquinone and dinitrobenzoate derivatives by a factor of 20, presumably due to a carrier effect in particle beam transport. A recent study suggested that the $\mathrm{NH}_{4} \mathrm{OAc}$ may act to help neutralize static charge produced by the nebulization process [15]

The anthraquinone derivatives were found to have an absorbance maximum at $257 \mathrm{~nm}$. The $\lambda_{\max }$ and extinction coefficient of the structurally similar $\mathrm{AQ}$ acid have been reported as $259 \mathrm{~nm}$ and 48,000 , respectively [16]. The anthraquinone derivatives did not fluoresce, which is consistent with a previous report [17]. Similarly, the application of $\mathrm{ACCl}$ derivatization for subsequent electrochemical detection appears to be of limited value. A recent study of the redox behavior of anthraquinone gave potentials of $-0.912 \mathrm{~V}$ for the reduction to the $\mathrm{AQ}^{-}$species and $-1.478 \mathrm{~V}$ to the fully reduced form $\mathrm{AQ}^{-2}$ [18]. Attempts to analyze the $A Q$ derivatives by electrochemical detection were not successful owing to the high potential necessary and high background at these voltages.

GC/MS analysis was demonstrated by using hexanol as the analyte. As expected, the derivative eluted from the column at a relatively high temperature (300 ${ }^{\circ} \mathrm{C}$ ). This is not surprising, because the same relative nonvolatility that gives the anthraquinone derivatives 
good transport properties through the particle beam interface also would require a high temperature to analyze by GC/MS. The parent compound anthraquinone, as well as methylaminoanthraquinone (MAAQ), have been studied by GC/MS $[19,20]$. Application of $\mathrm{ACCl}$ as a reagent for GC-NCI/MS would depend on the analyte of interest. Relatively low molecular weight alcohols may yield derivatives that would have sufficient volatility for GC analysis.

In comparison, pentafluorobenzoate esters of volatile alcohols are used routinely for GC-NCI analyses and exhibit prominent molecular anions [21]. A comparison of hexyl pentafluorobenzoate and hexyl anthraquinone carboxylate showed greater $\mathrm{GC}-\mathrm{NCI}$ detectability for the hexyl pentafluorobenzoate. This sensitivity enhancement was strongly temperature dependent, however: the anthraquinone derivative gained in sensitivity as the temperature increased, whereas the pentafluorobenzoate sensitivity decreased. An additional advantage of $\mathrm{ACCl}$ as a reagent for GC/ or LC/MS is that it imparts sufficient mass to the analyte to shift the molecular anion away from low mass chemical background. Finally, the NCI mass spectra of the derivatives in both particle beam and GC/MS analysis do not exhibit temperature-dependent fragmentation, which is an advantage in characterization of a variety of alcohols.

\section{Conclusions}

Anthraquinone-2-carbonyl chloride $(\mathrm{ACCl})$ has been synthesized and proven to be a reactive and sensitive reagent for the derivatization of primary and secondary alcohols with subsequent analysis by $\mathrm{PB}$ $\mathrm{LC}-\mathrm{NCI} / \mathrm{MS}$. The derivatives produced simple $\mathrm{NCI}$ mass spectra with all of the ion current residing in the molecular anion. No fragmentation occurred, even at ion source temperatures as high as $300{ }^{\circ} \mathrm{C}$. The anthraquinone electrophore was found to be greater than 100 times as sensitive in NCI versus EI. This sensitivity was demonstrated in SIM experiments that gave a limit of detection of $26 \mathrm{pg}$ for phenethyl anthraquinone carboxylate. The anthraquinone derivatives are favorable chromophores for detection by UV and, in the case of volatile alcohois, GC/MS.

\section{Acknowledgments}

The NIH/OSU student volunteer program is acknowledged for support of R. Worman. Amber I. Rouch assisted with catalyst evaluation and UV measurements. J. T. S. acknowledges Dr. Stephen Miller for many stimulating discussions that led to this work.

\section{References}

1. Hunt, D. F.; Stafford, G. C., Jr.; Crow, F. W.; Russell, J. W. Anal. Chem. 1976, 48, 2098.

2. Lewy, A. J.; Markey, S. P. Science 1978, 201, 741-743.

3. Williams, T. D.; Vachon, L.; Anderegg, R. J. Anal. Biochem. 1986, 153, 372-379.

4. Bergner, E. A.; Dougherty, R. C. Biol. Mass Spectrom. 1981, 8 , 204-207.

5. Knapp, D. R. Handbook of Analytical Derivatization Renctions; Wiley: New York, 1979.

6. Harrison, A. G. Chemical lonization Mass Spectrometry, 2nd ed.; CRC Press: Boca Raton, FL, 1992.

7. Schulz, R. C. Makromol. Chem. 1983, 184, 1143-1152.

8. Naito, K.; Miura, A.; Azuma, M. J. Am. Chem. Soc. 1991, 113, 6386-6395.

9. Boni, R. L.; Simpson, J. T.; Naritsin, D. B.; Saito, K.; Markey, S. P. Biol. Mass Spectrom. 1994, 23, 27-32.

10. The Merck Index, 10th ed.; Merck: Rahway, NJ, 1983.

11. Markey, S. P.; Markey, C. J.; Wang, T.-C. L.; Rodríguez, J. B. J. Am. Soc. Mass Spectrom. 1993, 4, 336-342.

12. Thompson, J. A.; Markey, S. P.; Fennessey, P. V. Clin. Chem. 1975, 21, 1892-1898.

13. Hartvig, P.; Strandberg, S.; Näslund, B. J. Chromatogr. 1976, $118,65-74$

14. Wallace, J. E.; Hamilton, H. E.; Goggin, L. K.; Blum, K. Anal. Chem. 1975, 47, 1516-1519.

15. Wilkes, J. G.; Freeman, J. P.; Heinze, T. M.; Lay, J. O., Jr. In 42nd ASMS Conference on Mass Spectrometry and Allied Topics; Chicago, IL, 1994, p 1023.

16. Okano, T.; Horie, T.; Ohno, J. Yakugaku Zasshi 1978, 98, 929-936.

17. Ushiki, H.; Okamoto, A.; Horie, K.; Mita, I. Bull. Chem. Soc. Ipn. 1983, 56, 3181-3182.

18. Revenga, J.; Rodriguez, F.; Tijero, J. I. Electrochem. Soc. 1994, 141, 330-333.

19. Verweij, A. M. A.; Lipman, P. J. L. J. Chromatogr. 1993, 653, 359-362.

20. Harger, W. P.; Helmig, D. Sci. Total Environ. 1994, 148, 11-21.

21. Blau, K.; Halket, J., Eds. Handbook of Derivatives for Chromatography, 2nd ed.; Wiley, New York, 1993. 